\title{
Towards Long-Term Collective Experiments
}

Florian Vaussard, Philippe Rétornaz, Steven Roelofsen, Michael Bonani, François Rey, and Francesco Mondada

\begin{abstract}
It is often challenging to manage the battery supply when dealing with a fleet of mobile robots during long experiments. If one uses classical recharge stations, then agents are immobilized during the whole recharge process. In this study, we present a novel approach that employs a battery pack swapping station. Batteries are charged in a rotating barrel, and the robots dock only for the time of the hot-swap process. We attained an unavailability time of only 40 seconds, with a success rate of $100 \%$ on a total of 46 trials. Experiments above 8 hours are performed in three arenas with different configurations, which proves the relevance of our approach.
\end{abstract}

\section{Introduction}

Collective robotics is a field generating a high academic interest. Using several robots has been proposed as an elegant way to solve complex problems, that are impossible for only one entity. We can cite, for example, experiments in collective gap crossing [8], collective construction [13], or cooperative localization and grasping of objects [3].

In practice, the main heel of collective robotics is the dependency on electric power, each autonomous entity usually having its own battery. The realisation of complex experiments often requires runs of several hours or even days. A classic solution is to use a charging station, which requires a downtime of the agents during the corresponding period. Often, to avoid this downtime, one person constantly maintains the setup. Otherwise, some laboratories have heavy installations consisting of a floor powering system, which is very expensive to build and to maintain. A new solution is needed to maintain an availability close to $100 \%$.

Florian Vaussard · Philippe Rétornaz · Steven Roelofsen · Michael Bonani · François Rey . Francesco Mondada

EPFL - STI - LSRO, Station 9, 1015 Lausanne, Switzerland

e-mail: florian.vaussard@epfl.ch 
In this study, we will demonstrate a novel solution for autonomous robots in the form of automatic hot-swapping of batteries. The design relevance is validated through a simulation, and the final prototype is tested under experimental conditions, totalling more than 24 hours of work with 3 robots operating simultaneously.

\section{Prior Works}

Batteries and other storage devices have always limited mobile devices. The problem of autonomously recharging a robot, or a fleet of robots, has already been extensively studied. Several designs have been considered and tested [2, 5, 9, 10, 11, 12]. The problem was also studied using Unmanned Aerial Vehicles (UAV) [4, 16]. However, with such an approach, the robots are unusable during the whole recharge process.

In the literature, battery swapping is often mentioned as a way to get rid of the charging time $[17,19]$. But practically speaking, few working implementations exist. To the best of our knowledge, authors of [18] are the first to realise a proof of concept using an autonomous robot. Their robot is able to enter the charging dock by following a magnetic tape, and the exchange process takes 45 seconds. However, the design is complex and is limited to two charging units. No other data is provided.

The work of [15] introduced the first prototype of a swapping system for an autonomous helicopter. It is able to recharge up to 8 batteries in parallel using related health management algorithms. No experiment yet proves the viability of the solution. Finally, several modules were developed and discussed in [14] for another UAV system, but no complete prototype is currently available.

Finally, some researchers used an arena with a conductive floor to provide a continuous power supply [6]. This is a big investment in terms of time and money, and requires hardware adaptations. Moreover, the floor has to be flat, which limits the relevance to a subset of experiments.

\section{Simulation}

We first modelled the battery swapping system to assess the relevance of our approach. Let us take $\mathrm{N}_{r}$ robots, each one having an autonomy of $\mathrm{T}_{r}$ seconds. We aim to build a charger capable of recharging $\mathrm{N}_{c}$ batteries in parallel, each battery taking $\mathrm{T}_{c}$ seconds to charge. At the equilibrium, the maximum number of robots we can sustain is approximated by the conservation law:

$$
N_{r}=N_{c} \cdot \frac{T_{c}}{T_{r}} .
$$

However, this is an idealization and does not account for the worst case scenario. To identify the requirements for the charger, we modelled $T_{r}$ and $T_{c}$ as Gaussian 


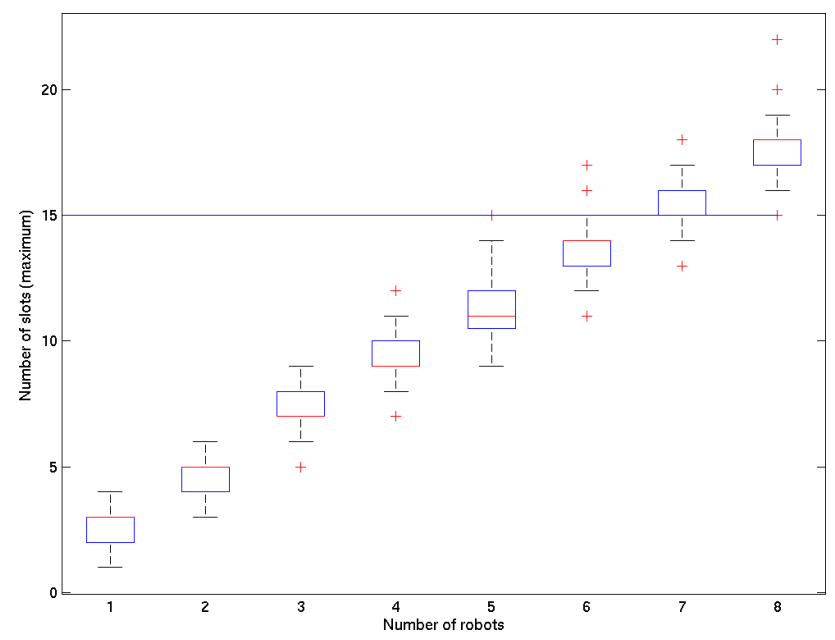

Fig. 1: Number of charging slots needed, with respect to the number of robots used during the experiment. The horizontal line is the targeted design (15 slots).

distributions. Parameters are given in Table 1 and are used to match the figures encountered in practice. A discrete time simulation is performed under Matlab and computes, at each time step, the maximum number of batteries simultaneously in charge. The result is shown in Fig. 1.

Table 1: Simulation parameters, modelled as Gaussian distributions.

\begin{tabular}{|c|c|c|c|}
\hline & & \multicolumn{2}{|c|}{ Time } \\
\hline & & $\begin{array}{l}\hat{\boldsymbol{T}} \\
(\mathrm{s})\end{array}$ & $\begin{array}{c}\boldsymbol{\sigma} \\
(\mathrm{s})\end{array}$ \\
\hline Autonomy & $T_{r}$ & 10800 & 3600 \\
\hline Recharge time & $T_{c}$ & 19800 & 1800 \\
\hline
\end{tabular}

For our first design, the target is fixed at 15 slots, due to the physical space constraints imposed by the size of the battery packs. According to this simulation, this choice allows up to 5 robots to run simultaneously. 


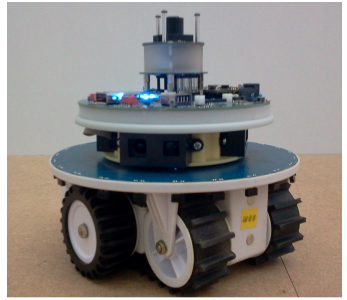

(a)

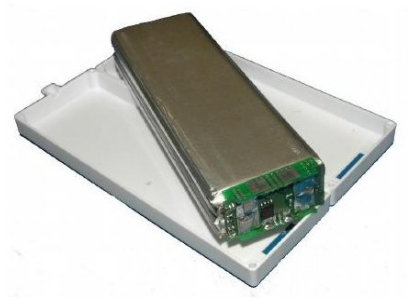

(b)

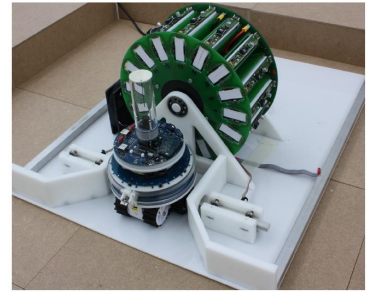

(c)

Fig. 2: Hardware design. (a) The marXbot robot. (b) The swappable battery pack (inside view). (c) The charging station with a docked marXbot.

\section{Hardware Design}

\subsection{Mobile Robotic Platform}

The robotic platform was designed with battery hot-swapping as a key feature from the beginning. We identified three requirements to ease long-term robotic swarm experiments: 1) online battery hot-swap, 2) automated battery exchange, and 3) compact design.

The first requirement is motivated as follows. If the robot must be switched off to swap its battery, then long-term experiments will be difficult. In most of the experiments, a wireless link to a supervision computer must be kept for logging or management purposes. The embedded computer must thus stay online for the whole experiment, keeping its state and all network connections opened.

The second requirement is of practical interest. If the experiments are run for several hours, or even days, then it is not efficient to manually swap the battery. Moreover, since we want to swap the battery while the system is running, we need a high level of determinism. A human is not able to achieve such reliability, especially in an experiment that lasts for several hours.

The third requirement seems obvious but is critical in small mobile robots. We cannot afford to have a big and bulky system, as it would increase the size and the weight of the robot. For collective experiments, it is a critical point, as many robots are used concurrently in a limited space.

The marXbot robot, pictured in Fig. 2a, was designed with these requirements in mind [1]. Both specific mechanical and electronic designs are needed to enable battery hot-swapping. 


\subsubsection{Mechanical Design}

The battery is located at the centre of the robot and is easily accessible from the backside. Moreover, the battery has a specific grabbing artefact that enables mechanical grasping. The battery is placed as low as possible, enhancing the stability by bringing down the centre of mass. The electrical contact is performed through lateral sliding contacts. When the battery is fully inserted, the contact is automatically established without requiring any specific connection mechanism.

\subsubsection{Electronic Design}

The electronics need to cope with the battery suddenly disappearing while running. This was resolved by using a backup supply. In our case, two $2.5 \mathrm{~V} / 10 \mathrm{~F}$ serially connected super-capacitors are embedded in the marXbot. They can power the vital subsystems, including the embedded computer, for 15 seconds. Two power buses are implemented throughout the robot. The first one is directly connected to the battery, typically for the motors and other non-critical electronics. The second bus is dynamically switched between the battery and the super-capacitor's supply.

A series of dedicated electronics are in charge of the switching by using an infrared sensor to detect the battery's removal. These electronics ensure a deterministic switching time, which provides a reliable power source. The system is fully autonomous and does not require any supervision.

The battery is a single cell 10 Ah lithium-polymer battery, as shown in Fig. $2 b$. The protection circuit is embedded in the plastic housing and the electrical connection is made using two gold-plated sideways printed circuit boards.

\subsection{Robotic Charger}

The robotic charger is wheel-shaped, with the charging slots distributed around the barrel. The main design goal was to fit as many batteries as possible in one station in order to sustain the operation of several robots at the same time. In order to let the experiment run unattended, the reliability was also an important driving criterion. The final station is shown in Fig. 2c.

\subsubsection{Mechanical Design}

The mechanical design is quite straightforward. A rotating barrel can hold and recharge 15 batteries in parallel. Two linear arms are placed on each side of the docking bay. When the robot arrives in front of the charger, the arms centre the robot and hold it firmly in place. A motorized gripper grasps the battery inside the robot, pulls it out of the mobile robot and brings it in the barrel. It then lets the barrel 
1)

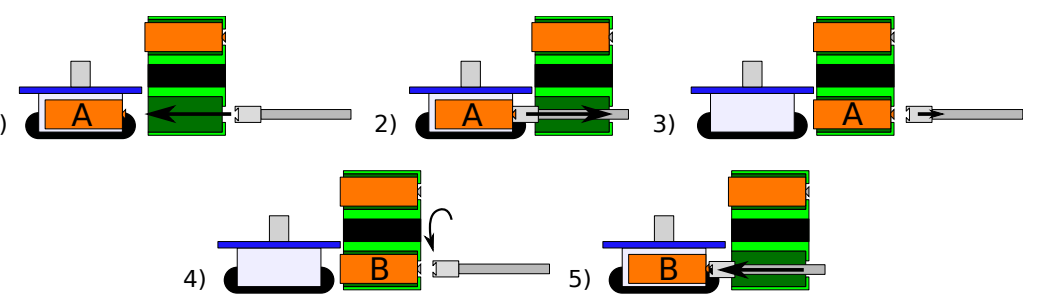

Fig. 3: Working principle of the battery exchanger.

rotate, and then pushes back a freshly charged battery. There are a total of 5 degrees of freedom. A diagram of the complete sequence is shown in Fig. 3.

\subsubsection{Electronic Design}

The 5 motors are controlled by several microcontrollers. The swapping state machine is entirely executed on the microcontrollers. The synchronization among the microcontrollers is performed through a CAN bus using the Aseba event-based framework [7]. Each slot has independent charging and monitoring electronics, ensuring a safe operation. Each battery is charged at $2.5 \mathrm{~A}$ under $4.2 \mathrm{~V}$. The total peak power consumption is about $160 \mathrm{~W}$.

\subsection{High Level Design}

The swapping station is connected to a supervision computer. Each robot taking part in the experiment is also connected to the computer through a Wi-Fi connection.

When a robot needs to get a fresh battery, it asks the supervision computer to get a lock on the charger. If no other robot is using the charger, then the computer grants the permission and the robot starts searching for the charger. If the charger is already in use, then the robot is put on a waiting list and has to wait until the supervisor gives it the lock.

As the arena used in this experiment is quite small, a search based on a wallfollowing method is used to find the swapping station. The method is able to detect loops, like isolated obstacles, as shown in Fig. 5a. This method proved to be robust in our case. The location of the charging bay is marked with an ISO-15693 RFID tag on the ground, so the robot can detect when it has to start the docking sequence.

Other methods should be envisioned in more complex environments, such as visual feature detection or range-based Simultaneous Localization And Mapping (SLAM). The marXbot has the necessary sensors and is directly suitable for both approaches. 


\section{Results}

We conducted two sets of experiments. The first one assesses the reliability and performance of the swapping mechanism. For this purpose, several consecutive swaps are conducted with the robot starting next to the station.

The second experiment is used to simulate a longer experiment, where several robots are covering the arena using a random walk. They autonomously go to the swapping station when the available energy reaches a low threshold.

Both experiments were run without any human interaction, except to start and stop the experiments.

\subsection{Battery Replacement}

In this experiment, we ran 24 battery swaps. The success rate was $100 \%$. The time required by each step of the process is shown in Fig. 4. The first step is the alignment and grasping of the robot by the station. During the second step, the robot runs without its battery on the backup supply. The charging station needs only 10 to 11 seconds to swap the battery. The entire process takes less than the 15 seconds provided by the embedded backup supply. The third step is the release of the robot, which is considerably faster than the others steps.

We can already draw a positive conclusion based on these results. The battery swap is indeed reliable and robust. The total time needed to change the battery is less than 40 seconds, while the robot runs only 10 seconds on the super-capacitors. This is fast enough to keep the ARM processor running and the Wi-Fi connection alive.

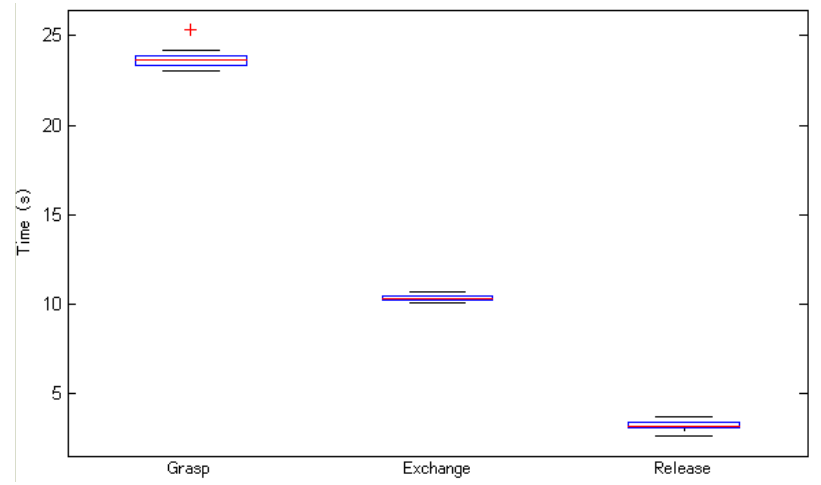

Fig. 4: Validation experiment: break down of the time required by each step of the battery replacement process. 


\subsection{Long Experiment}

We ran 3 long experiments, each one lasting more than 8 hours. During these experiments, three robots were moving randomly in a $2 \times 2 \mathrm{~m}$ arena. The configuration of the arena was changed between each experiment, as shown in Fig. 5a. In order to maximize the number of battery swaps, we decided to artificially limit the cut-off voltage to $3.6 \mathrm{~V}$.

We recorded the time needed to find the charger based on our naive wallfollowing approach (Fig. 5b). This method takes a considerable amount of time, in the order of several minutes. However, since the robots have an uptime of about 2-3 hours per battery, the 200 seconds needed to reach the charger are negligible. Thus, the wall-following method is perfectly justified in this situation, as it is robust and does not need any powerful computing resources.

Fig. 5c shows the results of the experiments. Each line corresponds to the voltage of individual robots. The robots successfully shared the same charger and were able to run for more than 8 hours without any failure. Each robot had its battery swapped several times and no power outages were experienced. In total, 22 successful swaps were performed.

\section{Conclusion}

We showed the possibility of autonomously hot-swapping the batteries of a small mobile robot. The achieved performance and reliability enables the use of small mobile robots during long term experiments. This opens the possibility of potential new research avenues on collective experiments.

This study shows that it is possible to have virtually infinite autonomy with only a minimal impact on the performed experiment. This is achieved through the careful design of the marXbot robot and the charger.

The next step is to use several chargers and a swarm of robots in a larger area. This would require better algorithms to share the resource and localize the charger. However, the proposed hardware remains perfectly valid.

Acknowledgements This research was supported by the Swiss National Science Foundation through the National Centre of Competence in Research Robotics. This work was also supported by the Swarmanoid and Ascens projects, both funded by the Future and Emerging Technologies programme (IST-FET) of the European Community, respectively under grants 022888 and 257414. The information provided is the sole responsibility of the authors and does not reflect the Community's opinion. The Community is not responsible for any use that might be made of data appearing in this publication. 
Towards Long-Term Collective Experiments

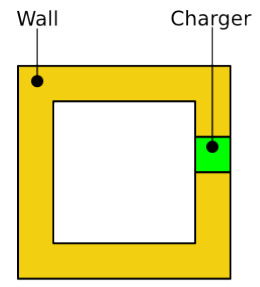

1)

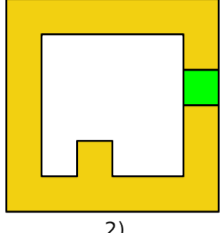

(a)

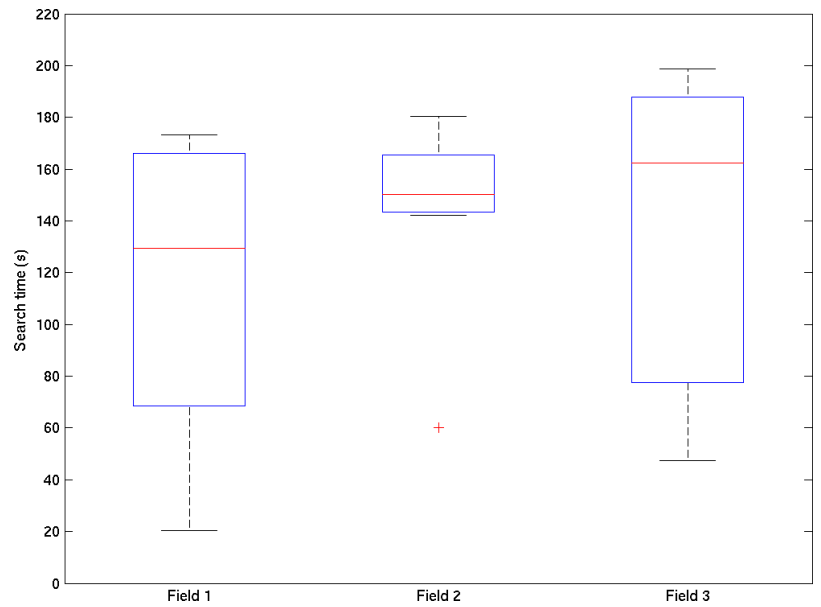

(b)
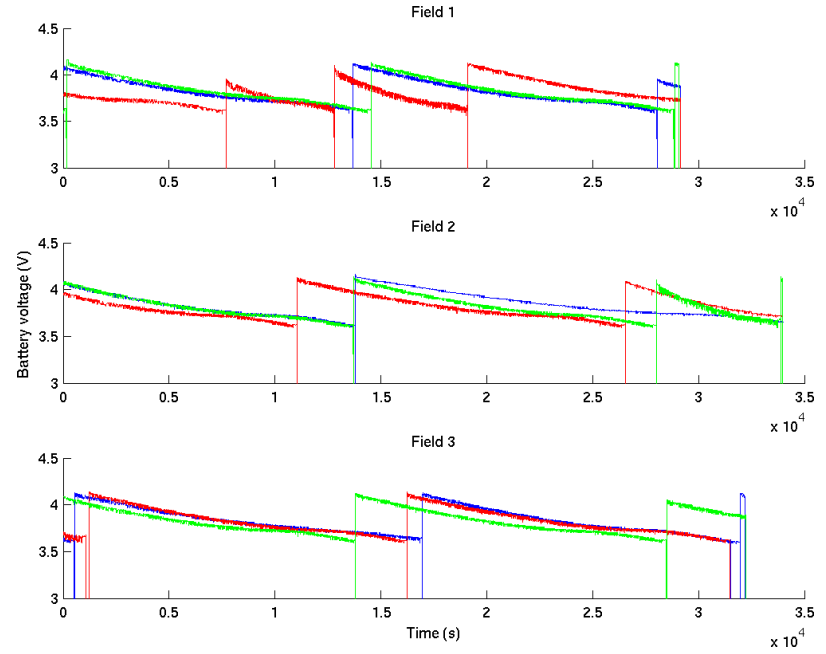

(c)

Fig. 5: The long-term experiments: (a) The three test fields. (b) Time to find and reach the charger on each field. (c) Voltage for each robot, as a function of the time and for each field. 


\section{References}

1. Bonani, M., Longchamp, V., Magnenat, S., Rétornaz, P., Burnier, D., Roulet, G., Vaussard, F., Bleuler, H., Mondada, F.: The marXbot, a miniature mobile robot opening new perspectives for the collective-robotic research. In: IROS. pp. 4187-4193. IEEE (2010)

2. Cassinis, R., Tampalini, F., Bartolini, P., Fedrigotti, R.: Docking and charging system for autonomous mobile robots. Department of Electronics for Automation, University of Brescia, Italy (2005)

3. Dorigo, M., Floreano, D., Gambardella, L., Mondada, F., Nolfi, S., Baaboura, T., Birattari, M., Bonani, M., Brambilla, M., Brutschy, A., et al.: Swarmanoid: a novel concept for the study of heterogeneous robotic swarms. Tech. rep., Technical Report TR/IRIDIA/2011-014, IRIDIA, Université Libre de Bruxelles, Brussels, Belgium (2011)

4. Kemper, F.P., Suzuki, K.A.O., Morrison, J.R.: UAV Consumable Replenishment: Design Concepts for Automated Service Stations. Journal of Intelligent and Robotic Systems 61(1-4), 369-397 (2011)

5. Kim, K., Choi, H., Yoon, S., Lee, K., Ryu, H., Woo, C., Kwak, Y.: Development of docking system for mobile robots using cheap infrared sensors. In: 1st International Conference on Sensing Technology, Palmerston North, New Zealand (2005)

6. Krieger, M.J.B., Billeter, J.B.: The call of duty: Self-organised task allocation in a population of up to twelve mobile robots. Robotics and Autonomous Systems 30(1-2), 65-84 (2000)

7. Magnenat, S., Rétornaz, P., Bonani, M., Longchamp, V., Mondada, F.: ASEBA: A Modular Architecture for Event-Based Control of Complex Robots. IEEE/ASME Transactions on Mechatronics (2010)

8. Mondada, F., Pettinaro, G., Guignard, A., Kwee, I., Floreano, D., Deneubourg, J., Nolfi, S., Gambardella, L., Dorigo, M.: SWARM-BOT: A new distributed robotic concept. Autonomous Robots 17(2), 193-221 (2004)

9. Parker, G., Georgescu, R., Northcutt, K.: Continuous power supply for a robot colony. In: Automation Congress, 2004. Proceedings. World. vol. 15, pp. 279-286. IEEE (2004)

10. Parker, G., Zbeda, R.: Controlled use of a robot colony power supply. In: Systems, Man and Cybernetics, 2005 IEEE International Conference on. vol. 4, pp. 3491-3496. IEEE (2005)

11. Silverman, M., Jung, B., Nies, D., Sukhatme, G.: Staying alive longer: Autonomous robot recharging put to the test. Center for Robotics and Embedded Systems (CRES) Technical Report CRES 3, 015 (2003)

12. Silverman, M.C., Nies, D., Jung, B., Sukhatme, G.S.: Staying Alive: A Docking Station for Autonomous Robot Recharging. In: ICRA. pp. 1050-1055. IEEE (2002)

13. Stroupe, A.W., Okon, A., Robinson, M.L., Huntsberger, T., Aghazarian, H., Baumgartner, E.T.: Sustainable cooperative robotic technologies for human and robotic outpost infrastructure construction and maintenance. Auton. Robots 20(2), 113-123 (2006)

14. Suzuki, K.A.O., Filho, P.K., Morrison, J.R.: Automatic Battery Replacement System for UAVs: Analysis and Design. Journal of Intelligent and Robotic Systems 65(1-4), 563-586 (2012)

15. Swieringa, K.A., Hanson, C.B., Richardson, J.R., White, J.D., Hasan, Z., Qian, E., Girard, A.: Autonomous battery swapping system for small-scale helicopters. In: ICRA. pp. 3335-3340. IEEE (2010)

16. Valenti, M., Bethke, B., Dale, D., Frank, A.A., McGrew, J.S., Ahrens, S., How, J.P., Vian, J.: The MIT Indoor Multi-Vehicle Flight Testbed. In: ICRA. pp. 2758-2759. IEEE (2007)

17. Wang, H., Xu, H., Jones, A.: Crucial Issues in Logistic Planning for Electric Vehicle Battery Application Service. In: Optoelectronics and Image Processing (ICOIP), 2010 International Conference on. vol. 1, pp. 362-366. IEEE (2010)

18. Wu, Y., Teng, M., Tsai, Y.: Robot docking station for automatic battery exchanging and charging. In: Robotics and Biomimetics, 2008. ROBIO 2008. IEEE International Conference on. pp. 1043-1046. IEEE (2009)

19. Yang, Y., Ding, X., Zhu, J.: Discuss Charging-Discharging and Sale Technology of the Electric Vehicle. Advanced Materials Research 347, 3908-3914 (2012) 